\title{
Synthesis and Polymerization of Aromatic Polyamide and Polyester Macromonomers
}

\author{
Mayumi Izawa, Sadaaki Nunomoto, ${ }^{\dagger}$ and Yusuke KawaKamI* \\ Faculty of Chemical Engineering, Toyama National College of Technology, \\ Hongo, Toyama 939, Japan \\ * Graduate School of Materials Science, \\ Japan Advanced Institute of Science and Technology, Hokuriku, \\ Asahidai 15, Tatsunokuchi, Ishikawa 923-12, Japan
}

(Received March 9, 1993)

\begin{abstract}
Polycondensation of a mixture of $p$ - and $m$-aminobenzoic acid in $7 / 3$ molar ratio, in the presence of phosphorus compounds, and $40 \mathrm{~mol} \%$ methacrylic acid (MA) or $p$-carboxystyrene (PCS) as a chain terminator gave end methacrylate-functionalized $(n=8)$ and styrene-functionalized $(n=6)$ polyamide macromonomers almost soluble in dimethylsulfoxide (DMSO) in $44 \%$ and $59 \%$ yield, respectively. Similar polycondensation of $p$-hydroxybenzoic acid in the presence of $60 \mathrm{~mol} \%$ p-carboxyphenyl methacrylate (CPM) or PCS as a chain terminator gave end methacrylatefunctionalized $(n=7)$ or styrene-functionalized $(n=6)$ polyester macromonomers almost soluble in 1-methyl-2-pyrrolidinone (NMP) in 36\% yield. Complete consumption of the macromonomers in the copolymerization with methyl methacrylate revealed that the polymerizable double bonds were almost quantitatively introduced into the macromonomers.
\end{abstract}

KEY WORDS Polycondensation / Polyamide Macromonomer / Polyester Macromonomer / Reactivity / Graft Copolymer / Thermal Analysis / Compatibility /

We have been interested in elucidating the relationship between chemical structure and the physical property of branched polymers. For example, we took advantage of the hydrophobic nature of polydimethylsiloxane branches of graft copolymers in modifying the surface property of base polymers by the addition of small amounts of the graft copolymer. $^{1,2}$ We also showed that the presence of short oligodimethylsiloxane as branches in polymer structure can enhance the permeability of gas through the polymer film. ${ }^{3-7}$

Since aromatic polyamides or polyesters have good mechanical and thermal properties, it is of interest to introduce aromatic polyamide or polyester as side-chains into main-chain of a vinyl polymer in order to improve the physical properties of the main-chain polymers. Meanwhile, macromonomers are versatile synthetic intermediate as monomers in synthesizing graft copolymers. They are introduced as the side-chains of graft copolymers. However, relatively few examples are seen in the literature about the macromonomer containing aromatic amide or ester function. ${ }^{8}$

It has been reported that various systems containing tri- or penta-valent phosphorus compounds, such as triphenyl phosphitepyridine, ${ }^{9,10}$ triphenylphosphine-hexachloroethane, ${ }^{11}$ phenyldichlorophosphine-lithium chloride, ${ }^{12}$ or hexachlorocyclotriphosphazene $^{13}$ are very effective for the synthesis of aromatic polyamide or polyesters from a

$\dagger$ To whom correspondence should be addressed. 
dicarboxylic acid and a diamine or diol.

This paper deals with the synthesis of aromatic polyamide and polyester macromonomers of well-controlled structure in the presence of phosphorus compounds as condensing agent, the polymerizabilities of these macromonomers, and the application of the macromonomers in improving the thermal property of vinyl polymers by the introduction of the polyamide or polyester macromonomer components as branches of the vinyl polymers.

\section{EXPERIMENTAL}

\section{Reagents}

$p$-Carboxystyrene was synthesized as reported. ${ }^{14,15}$ Magnesium was activated by grinding in the presence of small amounts of 1,2-dibromoethane. Strictly anhydrous condition is essential to avoid the formation of alcohol in carbonation of the Grignard reagent. ${ }^{16} p$-Carboxyphenyl methacrylate was synthesized by the reaction of $p$-hydroxybenzoic acid with excess methacrylic acid in the presence of phenyldichlorophosphinelithium chloride in pyridine. ${ }^{12}$ Other reagents were used after proper purification.

\section{Synthesis of Macromonomer}

The synthetic schemes are shown in Scheme 1.

In a typical procedure, $p$-aminobenzoic acid $(0.96 \mathrm{~g}, 7 \mathrm{mmol}), m$-aminobenzoic acid $(0.41 \mathrm{~g}$, $3 \mathrm{mmol})$, pyridine $(20 \mathrm{ml})$, triphenylphosphine $(2.62 \mathrm{~g}, 10 \mathrm{mmol})$, hexachloroethane $(2.37 \mathrm{~g}$, $10 \mathrm{mmol})$, and methacrylic acid (MA) $(0.34 \mathrm{~g}$, $4 \mathrm{mmol}$ ) were placed in a polymerization tube filled with nitrogen, and the mixture was heated at $80^{\circ} \mathrm{C}$ for $3 \mathrm{~h}$. The reaction product was poured into methanol $(100 \mathrm{ml})$ to terminate the polymerization. The precipitate was collected by centrifugation, washed four times with methanol ( $50 \mathrm{ml}$ each), and dried in a vacuum desiccator. The dried product was pulverized and immersed in $10 \% \mathrm{NaCl}$ aqueous solution $(50 \mathrm{ml})$ to remove pyridinium chloride. The

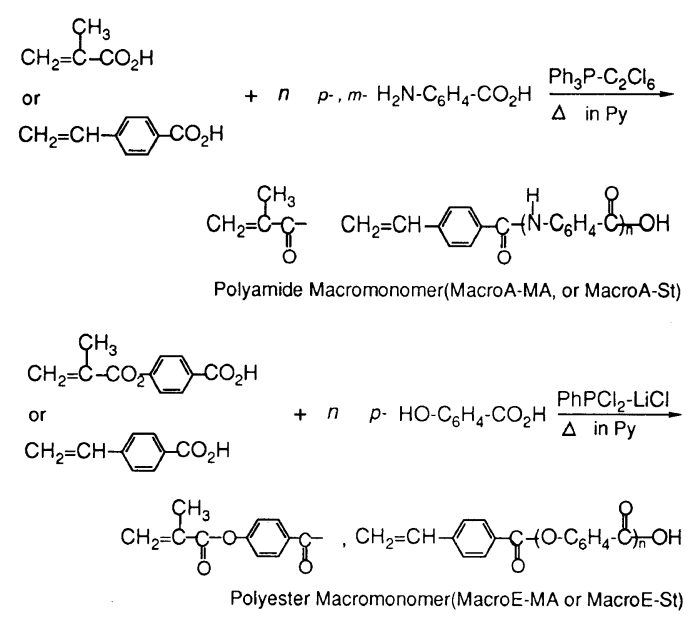

Scheme 1. Synthesis of macromonomers.

solid was separated by suction filtration, washed three times with the $\mathrm{NaCl}$ solution and water $(100 \mathrm{ml}$ each) successively, followed by three times with methanol $(50 \mathrm{ml}$ each), and dried in a vacuum desiccator.

One gram of the product (crude macromonomer) was dissolved in DMSO $(20 \mathrm{ml})$, the insoluble fraction was removed by centrifugation $(15000 \mathrm{rpm})$, and the end methacrylatefunctionalized polyamide macromonomer (MacroAM) was recovered from DMSO soluble fraction by precipitating into methanol.

Polyester macromonomer was similarly synthesized in the presence of CPM or PCS and phenyldichlorophosphine-lithium chloride in pyridine. One gram of the crude macromonomer was dissolved in NMP $(10 \mathrm{ml})$, centrifuged, and the purified macromonomer was obtained from NMP soluble fraction by precipitating into methanol. The yields of the macromonomers are shown in Table I.

End methacrylate-functionalized polyamide macromonomers is abbreviated as macroAM, and end styrene-functionalized as macroAS. End methacrylate- and styrene-functionalized polyester macromonomers are abbreviated as macroEM and macroES. 
Analysis of the Macromonomers and Copolymers

The molecular weights of the macromonomers soluble in DMSO (polyamide macromonomer) or NMP (polyester macromonomer) and their distributions were estimated by the use of a Waters GPC model 244 with Shodex columns AD-80M (molecular weight of exclusion limit for poly(ethylene glycol) $=2 \times 10^{7}, 60 \mathrm{~cm}$ ) and KD 802.5 (molecular weight of exclusion limit for poly(ethylene glycol) $=2 \times 10^{4}, 30 \mathrm{~cm}$ ) using poly(ethylene glycol) as the standard in dimethylformamide (DMF) containing $0.05 \mathrm{wt} \%$ lithium chloride and phosphoric acid (flow rate $=0.8 \mathrm{ml} \mathrm{min}^{-1}$ ) at $23^{\circ} \mathrm{C}$. The number average degree of polymerization of the macromonomers was also estimated by ${ }^{1} \mathrm{H}$ NMR spectroscopy using the spectral area ratio of $\mathrm{CH}_{3}-$ or $\mathrm{CH}_{2}=\mathrm{C} \underline{\mathrm{H}}-$ to $-\mathrm{C}_{6} \underline{\mathrm{H}}_{4}-$ for methacrylate-functionalized macromonomer (macroAM or macroEM) and styrene-functionalized macromonomer (macroAS or macroES), respectively. The copolymers were analyzed under same conditions by using a GPC. The composition of the copolymers was analyzed by ${ }^{1} \mathrm{H}$ NMR spectroscopy.

\section{Functionality of Macromonomer}

The functionality of the polymerizable group of the macromonomers was estimated by homopolymerization or copolymerization with methyl methacrylate (MMA) or styrene.

In the copolymerization, approximately $0.5 \mathrm{~g}$ $(0.50 \mathrm{mmol})$ of a macromonomer and $15 \mathrm{mmol}$ of a comonomer were polymerized with $0.05 \mathrm{~g}$ $(2 \mathrm{~mol} \%)$ of AIBN to the monomers in $10 \mathrm{ml}$ of DMSO (polyamide macromonomer) or NMP (polyester macromonomer) under nitrogen at $50^{\circ} \mathrm{C}$ for $20 \mathrm{~h}$.

In the separation of a graft copolymer from polyamide macromonomer, the reaction product was poured into $100 \mathrm{ml}$ of methanol, centrifuged, washed with methanol, and dried in a vacuum desiccator. The product was extracted with chloroform (in case of copo- lymerization with MMA) or benzene (in case of copolymerization with styrene) in order to remove the homopolymer of the comonomer. The fraction remaining after the extraction was considered to be the copolymer.

In the separation of a graft copolymer from polyester macromonomer, the crude reaction product was recovered by pouring into $100 \mathrm{ml}$ of methanol, and the formed solid was washed with methanol and dried in a vacuum desiccator.

In case of the copolymerization with styrene, the crude product was dissolved in NMP and poured into benzene-methanol $(6: 1)$ in order to remove the unreacted macromonomer as insoluble fraction. The soluble fraction was concentrated by the evaporation of the solvent, and treated with cyclohexane at $35^{\circ} \mathrm{C}$ in order to remove the homopolymer of the comonomer, namely, polystyrene, if any. The cyclohexane insoluble fraction was considered to be the graft copolymer.

In case of copolymerization with MMA, the product was poured into chloroform-methanol (55:15) after dissolved in NMP in order to remove the unreacted macromonomer as the insoluble fraction. The soluble fraction was concentrated, and treated with carbon tetrachloride at $30^{\circ} \mathrm{C}$. The insoluble fraction in the treatment with carbon tetrachloride was considered to be the graft copolymer.

Typical purification procedures of the copolymerization products (macroEM-Styrene: No 5; macroES-MMA: No 8 of Table II) are shown in Scheme 2. The procedure was repeated if necessary.

\section{Thermal Analysis}

DSC analyses of the macromonomers and copolymers were carried out on a SEIKO thermal analysis system model SSC 5500 equipped with DSC 100 with the heating rate of $10^{\circ} \mathrm{C} \mathrm{min}-1$. 

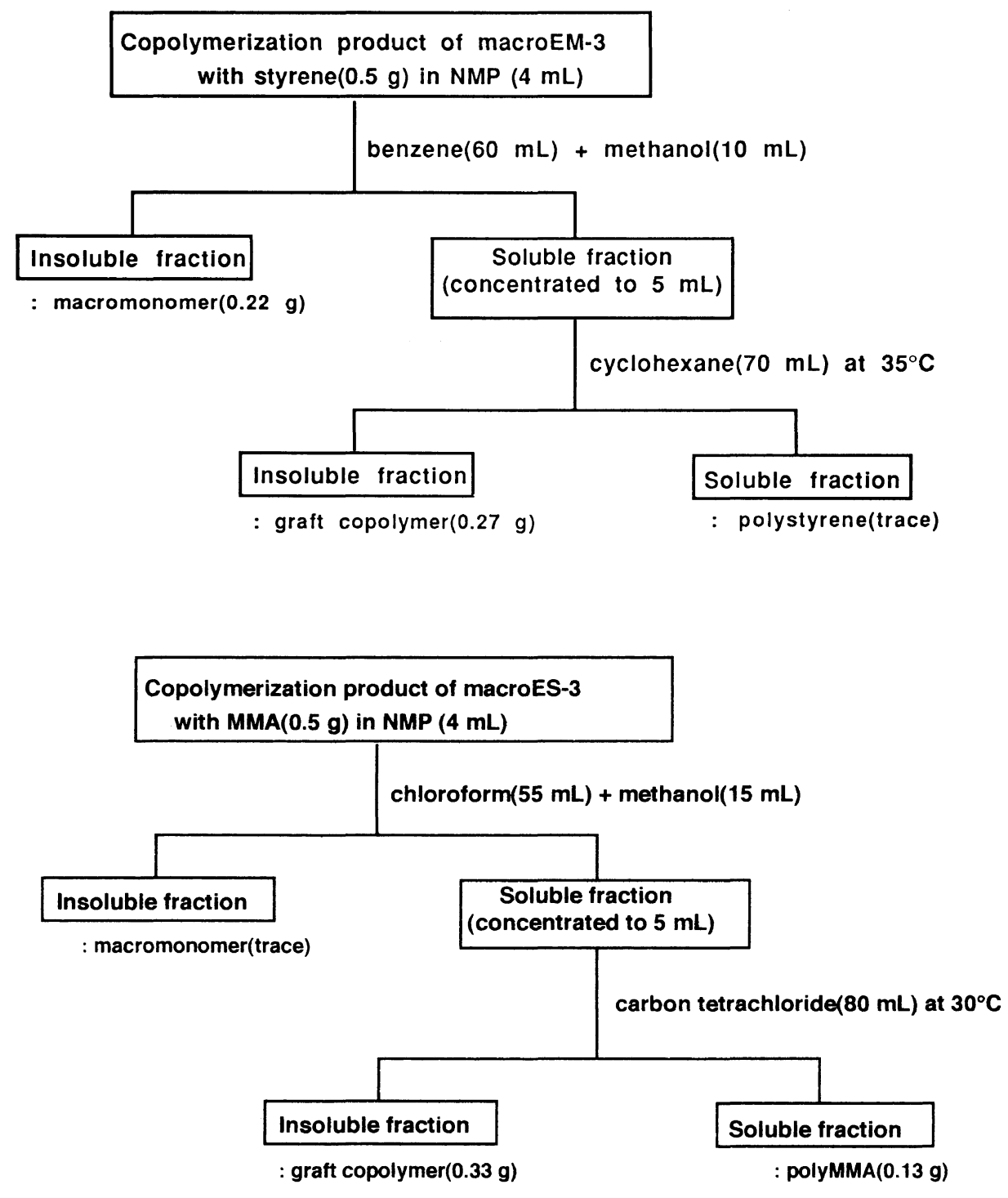

Scheme 2. Procedure of isolation of graft copolymer.

\section{RESULTS AND DISCUSSION}

Activation of the polycondensation of $p$-aminobenzoic acid by triphenylphosphinehexachloroethane ${ }^{11}$ used here, is superior to that by other phosphorus systems, such as phenyldichlorophosphine-pyridine-lithium chloride ${ }^{12}$ which was used in the synthesis of polyester macromonomer. The results of polycondensation of $p$-and $m$-mixture $(7: 3)$ of aminobenzoic acid in the presence of MA (AM series) or PCS (AS series) are shown in Table I.

Control A experiment shows that polyamide was produced in high yield with this combination of the reagents. The methanol insoluble fraction decreased with the increase in the concentration of MA from 0 to $60 \mathrm{~mol} \%$. Corresponding to this, the product became more soluble in polar solvents like DMSO, DMF, and NMP. Macromonomers almost 
Table I. Synthesis of polyamide and polyester macromonomers ${ }^{\mathrm{a}}$

\begin{tabular}{|c|c|c|c|c|c|c|c|c|}
\hline \multirow{2}{*}{ No. } & \multirow{2}{*}{$\frac{\text { MA or PCS }}{\mathrm{mol} \%}$} & \multirow{2}{*}{$\frac{\text { Yield }^{\mathrm{b}}}{\%}$} & \multicolumn{2}{|c|}{ Solubility $^{\mathrm{c}}$} & \multirow{2}{*}{$\begin{array}{c}{ }^{1} \mathrm{H} \text { NMR } \\
\text { DP }, n^{\mathrm{d}}\end{array}$} & \multicolumn{3}{|c|}{ Molecular weight (GPC) $)^{\mathbf{e}}$} \\
\hline & & & Soluble & Insoluble & & $\bar{M}_{w} \times 10^{2}$ & $\bar{M}_{n} \times 10^{2}$ & $\bar{M}_{w} / \bar{M}_{n}$ \\
\hline Control A & 0 & 82 & 59 & 41 & - & - & - & - \\
\hline AM-1 & 20 & 70 & 85 & 15 & - & - & - & - \\
\hline AM-2 & 40 & 44 & 91 & 9 & 8 & 13.5 & 8.65 & 1.56 \\
\hline AM-3 & 60 & 36 & - & - & - & - & - & - \\
\hline AS-1 & 20 & 63 & 75 & 25 & - & - & - & - \\
\hline AS-2 & 40 & 59 & 88 & 12 & 6 & 11.3 & 6.40 & 1.77 \\
\hline AS-3 & 60 & 40 & 91 & 9 & - & - & - & - \\
\hline AS-4 & 120 & 20 & 95 & 5 & 4 & 5.19 & 3.94 & 1.32 \\
\hline Control E & 0 & 90 & 52 & 48 & - & - & - & - \\
\hline EM-3 & 60 & 36 & 72 & 28 & 7 & 8.65 & 5.54 & 1.56 \\
\hline ES-3 & 60 & 36 & 81 & 19 & 6 & 7.80 & 5.80 & 1.34 \\
\hline
\end{tabular}

a Monomer: $p$ - and $m$-aminobenzoic acid $(70: 30)=10 \mathrm{mmol} ; \mathrm{Ph}_{3} \mathrm{P}-\mathrm{C}_{2} \mathrm{Cl}_{6}=10 \mathrm{mmol}$; pyridine $=20 \mathrm{ml}$; time, $3 \mathrm{~h}$; temp, $80^{\circ} \mathrm{C}$ for AM-series, $50^{\circ} \mathrm{C}$ for AS-series. MA (AM-series) or PCS (AS-series) was used. Monomer: $p$-hydroxybenzoic acid $=10 \mathrm{mmol} ; \mathrm{PhPCl}_{2}-\mathrm{LiCl}=10 \mathrm{mmol}$; pyridine $=20 \mathrm{ml}$; time, $4 \mathrm{~h}$, temp, $50^{\circ} \mathrm{C}$ for $\mathrm{E}$-series. CPM (EM-series) or PCS (ES-series) was used.

b Methanol insoluble fraction based on the starting materials (MA or PCS + aminobenzoic acid or $p$-hydroxybenzoic acid).

c One gram of the crude macromonomer was dissolved in DMSO (20 ml) for AM- and AS-series or NMP (10 ml) for E-series, and the insoluble fraction was separated by centrifugation $(15000 \mathrm{rpm})$.

${ }^{d}$ Degree of polymerization by ${ }^{1} \mathrm{H}$ NMR.

e Molecular weights of DMSO soluble fraction of polyamide macromonomer and NMP soluble fraction of polyester macromonomer using PEG standard.

soluble in DMSO were produced in $44 \%$ yield in the presence of $40 \mathrm{~mol} \%$ of MA. The molecular weight of the macromonomer soluble in DMSO determined by GPC or ${ }^{1} \mathrm{H}$ NMR also decreased with the increase in the concentration of MA as the chain terminator. NMR spectrum indicates that the number average degree of polymerization of polyamide repeating units in methacrylate-functionalized macromonomer (macroAM-2) is 8 , corresponding to molecular weight of 1038 . The number average molecular weight estimated by GPC was 865 . The polydispersity was 1.56 .

A macromonomer almost soluble in DMSO was produced in 59\% yield in the presence of $40 \mathrm{~mol} \%$ PCS. The number average degree of polymerization of the macromonomer (macroAS-2) is estimated to be 6 , corresponding to molecular weight of 862 . The molecular weight by GPC was 640 , again a little smaller than the value by ${ }^{1} \mathrm{H}$ NMR. Macromonomer obtained in the presence of $120 \mathrm{~mol} \%$ PCS had $n=4$ by NMR.

The results of polycondensation of $p$ hydroxybenzoic acid in the presence of CPM (EM series) or PCS (ES series) are also shown in Table I. Control E experiment shows that polyester was produced in high yield under the reaction condition. The methanol insoluble fraction decreased with the increase in the concentration of CPM from 0 to $60 \mathrm{~mol} \%$. Corresponding to this, the product became more soluble in NMP. Macromonomers almost soluble in NMP were produced in $36 \%$ yield in the presence of $60 \mathrm{~mol} \%$ of CPM. The molecular weight of the macromonomer soluble in NMP determined by GPC or ${ }^{1} \mathrm{H}$ NMR also decreased with the increase in the concentration of CPM as the chain terminator. NMR spectrum indicates that the number average degree of polymerization of polyester repeating units in methacrylate-functionalized 
Table II. Copolymerization of aromatic macromonomers ${ }^{a}$

\begin{tabular}{|c|c|c|c|c|c|c|c|}
\hline \multirow{2}{*}{ No. } & \multirow{2}{*}{$\frac{\text { Macromonomer }}{\mathrm{mol} \%}$} & \multirow{2}{*}{$\frac{\text { Comonomer }}{\text { mol } \%}$} & \multirow{2}{*}{$\frac{\text { Yield }^{\mathrm{b}}}{\%}$} & \multirow{2}{*}{$\begin{array}{c}\text { Composition }^{\mathrm{b}} \\
\text { mol } \% \text { of macromonomer } \\
\text { by }{ }^{1} \mathrm{H} \text { NMR }\end{array}$} & \multicolumn{2}{|c|}{ Molecular weight $(\mathrm{GPC})^{\mathrm{c}}$} & \multirow{2}{*}{$\frac{T_{\mathrm{g}}}{{ }^{\circ} \mathrm{C}}$} \\
\hline & & & & & $\begin{array}{l}\bar{M}_{w} \\
\times 10^{3}\end{array}$ & $\bar{M}_{w} / \bar{M}_{n}$ & \\
\hline 1 & $\begin{array}{l}\text { macroAM-2 } \\
3\end{array}$ & $\begin{array}{l}\text { St } \\
97\end{array}$ & $50(65)$ & $7(6)$ & 20.2 & 1.89 & 101.3 \\
\hline 2 & $\begin{array}{c}\text { macroAM-2 } \\
3\end{array}$ & $\begin{array}{l}\text { MMA } \\
97\end{array}$ & $88(93)$ & $2(1)$ & 85.9 & 2.03 & 130.3 \\
\hline 3 & $\underset{4}{\operatorname{macroAS}-2}$ & $\begin{array}{l}\text { St } \\
96\end{array}$ & $54(65)$ & $7(6)$ & 42.5 & 2.08 & 108.9 \\
\hline 4 & $\begin{array}{c}\text { macroAS-2 } \\
4\end{array}$ & $\begin{array}{l}\text { MMA } \\
96\end{array}$ & 87 (92) & $4(3)$ & 132.0 & 2.37 & - \\
\hline 5 & $\begin{array}{c}\text { macroEM-3 } \\
3\end{array}$ & $\begin{array}{l}\text { St } \\
97\end{array}$ & $29(45)$ & $3(6)$ & 32.8 & 2.15 & - \\
\hline 6 & $\begin{array}{c}\text { macroEM-3 } \\
3\end{array}$ & $\begin{array}{c}\text { MMA } \\
97\end{array}$ & $65(85)$ & $3(6)$ & 61.3 & 1.85 & - \\
\hline 7 & $\begin{array}{c}\text { macroES-3 } \\
4\end{array}$ & $\begin{array}{l}\text { St } \\
96\end{array}$ & $38(51)$ & $3(7)$ & 28.9 & 2.02 & - \\
\hline 8 & $\begin{array}{c}\text { macroES-3 } \\
4\end{array}$ & $\begin{array}{l}\text { MMA } \\
96\end{array}$ & $80(83)$ & $3(4)$ & 78.8 & 1.96 & - \\
\hline
\end{tabular}

a Reaction conditions: see the text.

b Yield and composition of the remaining fraction after the extraction with benzene (No. 1 and 3), or with chloroform (No. 2 and 4) for the copolymer of polyamide macromonomer. Yield and composition of the insoluble fraction after the purification by reprecipitation (No. 5 and 7, cyclohexane; No. 6 and 8, carbon tetrachloride) for the copolymer of polyester macromonomer. Values in the parentheses are those of crude methanol insoluble fraction.

c Molecular weight of copolymer peak determined by GPC using PEG standard.

macromonomer (macroEM-3) is 7, corresponding to molecular weight of 1046. The number average molecular weight estimated by GPC was 554. The polydispersity was 1.56 .

A macromonomer almost soluble in NMP was produced in $36 \%$ yield in the presence of $60 \mathrm{~mol} \% \mathrm{PCS}$. The number average degree of polymerization of the macromonomer (macroES-3) is estimated to be 6 , corresponding to molecular weight of 868 . The molecular weight by GPC was 580 , again a little smaller than the value by ${ }^{1} \mathrm{H}$ NMR.

The higher number average molecular weight by ${ }^{1} \mathrm{H}$ NMR than that by GPC might reflect incomplete introduction of polymerizable functional groups into the macromonomer. The functionality of macromonomers was further investigated by studying the polymerizability of the macromonomers.
Double bonds of both styrene- and methacrylate-functionalized macromonomers disappeared completely, as shown by ${ }^{1} \mathrm{H}$ NMR spectroscopy.

The results of copolymerization of the macromonomers (macroAM-2, macroEM-3 and macroAS-2, macroES-3) with methyl methacrylate (MMA) or styrene $(\mathrm{St})$ as the comonomer to produce a graft copolymer are shown in Table II.

In the copolymerization of polyamide macromonomer, the methanol and chloroform (or benzene) soluble fraction of the copolymerization with MMA (or styrene) does not show any indication of the existence of macromonomer residue, which indicates that the macromonomer has been incorporated in the copolymer, or remained unreacted in the copolymerization mixture. 


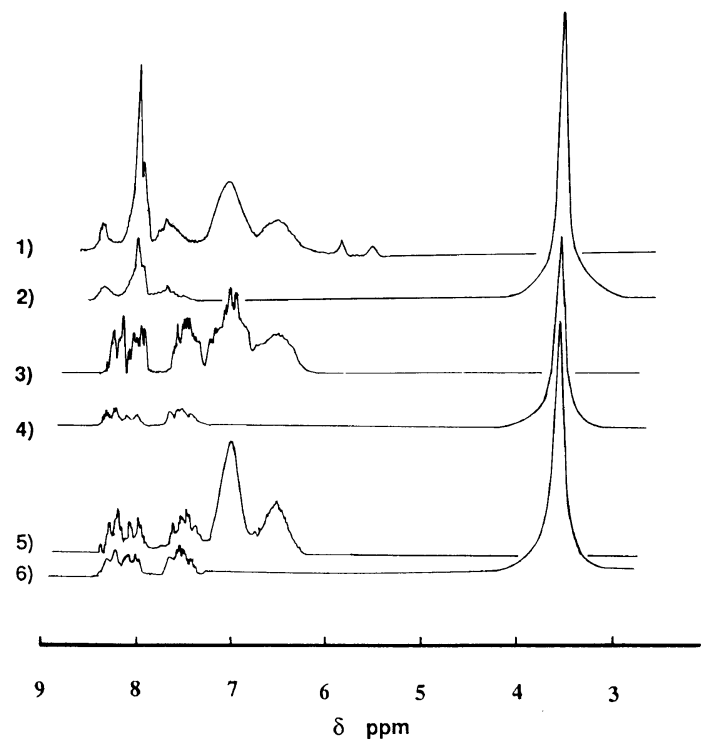

Figure 1. ${ }^{1} \mathrm{H}$ NMR spectra of aromatic and methoxy regions of copolymers of 1) macroAM-2 with styrene, 2) macroAM-2 with MMA, 3) macroEM-3 with styrene, 4) macroEM-3 with MMA, 5) macroES-3 with styrene, and 6) macroES-3 with MMA.

In the copolymerization with styrene, the yield of the methanol insoluble fraction is only $65 \%$, irrespective of the polymerizable functional group of the macromonomer, and the apparent concentration of macromonomer in the crude product, as determined by ${ }^{1} \mathrm{H}$ NMR (No. 1 of Figure 1; No. 1 and 3 of Table II), was higher than that in feed. This apparently shows that macromonomer has a higher reactivity in the copolymerization with styrene. However, not only the existence of double bond of the macromonomer in the NMR spectrum (No.1 of Figure 1), but also the GPC traces of the copolymer with styrene shown in Figure 2 (No. 1 and 3) indicate the presence of unreacted macromonomer even after the extraction of the copolymerization product with benzene. The NMR data cannot be strictly interpreted in these cases. The content of macromonomer even increased by the extraction procedure. Since the macromonomers have lower solubility in the solvents than the copolymers, the macromonomers could not be removed by

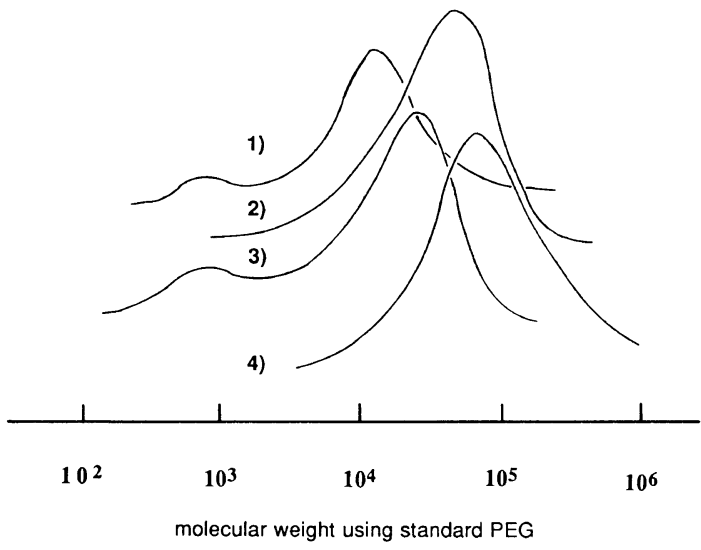

Figure 2. GPC chromatograms of copolymerization products of 1) macroAM-2 with styrene, 2) macroAM-2 with MMA, 3) macroAS-2 with styrene, and 4) macroAS-2 with MMA.

reprecipitation or extraction, which might have resulted in the apparently higher concentration of the macromonomers in the copolymerization product when determined by ${ }^{1} \mathrm{H}$ NMR. Styrene seems to be reluctant to participate in the copolymerization. Although the reason is not clear, the difficulty of the access of styrene to the polymerizable group of the polyamide macromonomer might be the reason of the low reactivity of styrene.

No macromonomer remained in the chromatogram of the copolymerization product with MMA after the extraction procedure (No. 2 and 4 of Figure 2). ${ }^{1} \mathrm{H}$ NMR spectrum in this case gives a definite copolymer composition (No. 2 of Figure 1; No. 2 and 4 of Table II). The macromonomers seem to exhibit a similar reactivity with methyl methacrylate. The complete disappearance of the macromonomers in the copolymerization with MMA assures the quantitative introduction of the polymerizable double bond to the polyamide macromonomers.

Similar trend was also observed in the copolymerization of polyester macromonomer with styrene or methyl methacrylate. The yield was low in the copolymerization with styrene. In the GPC of the product from run No. 5 of 


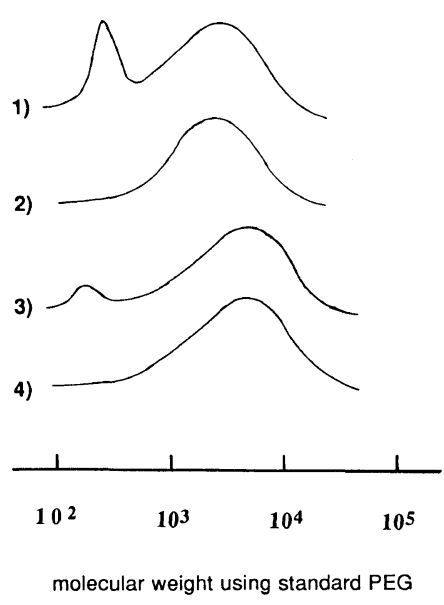

Figure 3. GPC chromatograms of copolymerization products of 1) macroEM-3 with styrene as precipitated in methanol, 2) macroEM-3 with styrene after purification, 3) macroES-3 with MMA as precipitated in methanol, and 4) macroES-3 with MMA after purification.

Table II, shown as No.1 of Figure 3, considerable amounts of unreacted macromonomer were contained, which could be removed by isolation procedure of the graft copolymer shown in Scheme 2 (No. 2 of Figure 3 ), resulted in rather poor yield of the graft copolymer. Similar phenomena were observed in the copolymerization of styrene-functionalized polyester macromonomer (No. 7 of Table II) with styrene.

Slightly remaining macromonomer in the crude copolymerization product with methyl methacrylate (No. 8 of Table II: No. 3 of Figure 3) was completely removed by isolation procedure (No. 4 of Figure 3), which indicates that this system gave the true graft copolymer in good yield. Methacrylate-functionalized polyester macromonomer gave similar results (No. 6 of Table II).

${ }^{1} \mathrm{H}$ NMR spectra of other graft copolymers from polyester macromonomers are also shown in Figure 1.

DSC curves of the graft copolymers from polyamide macromonomer are shown in Figure 4.

The polyamide shows a primary transition

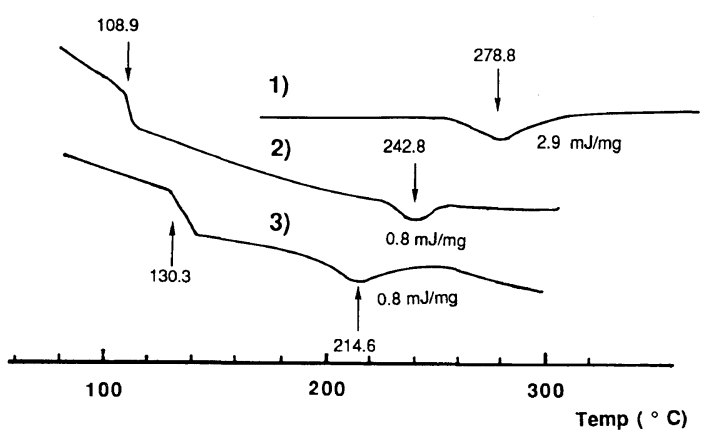

Figure 4. DSC curves of polyamide and graft copolymers: 1) polyamide ( $p-: m-=7: 3)$; 2 ) graft copolymer No. 3 of Table II; 3) graft copolymer No. 2 of Table II.

of heat capacity $2.9 \mathrm{~mJ} \mathrm{mg}^{-1}$ at $279^{\circ} \mathrm{C}$ which may be ascribable to partial crystallization of the polymer. The graft copolymer with the polystyrene backbone (No. 3 of Table II) had $T_{\mathrm{g}}=109^{\circ} \mathrm{C}$ which is close to the $T_{\mathrm{g}}$ of polystyrene. By contrast, the graft copolymer with the polymethacrylate backbone (No. 2 of Table II) had a markedly higher $T_{\mathrm{g}}=130^{\circ} \mathrm{C}$ as compared with the $T_{\mathrm{g}}$ of PMMA. Since the main-chain and the side-chain components are very incompatible in the graft copolymers with the polystyrene backbone, the property of main-chain polymer was not influenced by the side-chains. In the case of the graft copolymer with polymethacrylate backbone, the mainchain and the side-chains can interact through the polar amide and ester groups, which will result in the increase of $T_{\mathrm{g}}$. In both cases, partial crystallization of the polyamide side chains seems to be disturbed by the main-chain as seen by the decrease in the heat capacity of the transition above $215^{\circ} \mathrm{C}$.

The graft copolymer from polyester macromonomer did not show glass transition temperature at the temperature range of that of poly(methyl methacrylate) or polystyrene. The glass transition temperature might have been elevated to higher temperature. These facts indicate the effectiveness of the modification of the thermal property of the base polymer by side-chains compatible with the main-chain. 
Acknowledgments. Financial supports from a Grant-in-Aid for Scientific Research on Priority Areas, New Functionality MaterialsDesign, Preparation, and Control (04205065) from the Ministry of Education, Science, and Culture of Japan is gratefully acknowledged.

\section{REFERENCES}

1. Y. Kawakami, R. A. N. Murthy, and Y. Yamashita, Makromol. Chem., 185, 9 (1984).

2. Y. Kawakami, T. Aoki, and Y. Yamashita, Macromolecules, 18, 580 (1985).

3. Y. Kawakami, T. Aoki, H. Hisada, Y. Yamamura, and Y. Yamashita, Polym. Comm., 26, 133 (1985).

4. Y. Kawakami, H. Karasawa, T. Aoki, Y. Yamamura, H. Hisada, and Y. Yamashita, Polym. J., 17, 1159 (1985).

5. Y. Kawakami, H. Karasawa, H. Kamiya, T. Aoki, and Y. Yamashita, Polym. J., 18, 237 (1986).

6. Y. Kawakami, T. Sugisaka, and Y. Yamashita,
Polym. J., 20, 685 (1988).

7. Y. Kawakami and T. Sugisaka, J. Memb. Sci., 50, 189 (1990).

8. M. Niwa and N. Higashi, Macromolecules, 21, 1191 (1988).

9. N. Yamazaki and F. Higashi, Adv. Polym. Sci., 38, 1 (1981).

10. S. M. Aharoni, W. B. Hammomd, J. S. Szobota, and D. Masilamani, J. Polym. Sci.-Polym. Chem. Ed., 22, 2579 (1984).

11. G. C. Wu, H. Tanaka, K. Sanui, and N. Ogata, Polym. J., 14, 571 (1982).

12. F. Higashi, N. Kokubo, and M. Goto, J. Polym. Sci.-Polym. Chem. Ed., 18, 2879 (1980).

13. F. Higashi, K. Kubota, M. Sekizuka, and M. Higashi, J. Polym. Sci.-Polym. Chem. Ed., 19, 2681 (1981).

14. G. B. Bachman, C. L. Carlson, and M. Robinson, $J$. Am. Chem. Soc., 73, 1964 (1951).

15. J. R. Leebrick and H. E. Ramsden, J. Org. Chem., 23, 935 (1958).

16. R. P. Quirk, J. Yin, and L. J. Fetters, Macromolecules, 22, 85 (1989). 